\title{
Real-time Al prediction for major adverse cardiac events in emergency department patients with chest pain
}

Pei-l Zhang ${ }^{1 \dagger}$, Chien-Chin Hsu ${ }^{2,3+}$, Yuan Kao ${ }^{2,4}$, Chia-Jung Chen ${ }^{5}$, Ya-Wei Kuo ${ }^{6}$, Shu-Lien Hsu' ${ }^{7}$, Tzu-Lan Liu ${ }^{5}$, Hung-Jung Lin ${ }^{2,8}$, Jhi-Joung Wang ${ }^{9,10}$, Chung-Feng Liu ${ }^{9}$ and Chien-Cheng Huang ${ }^{2,11,12^{*}}$ (D)

\begin{abstract}
Background: A big-data-driven and artificial intelligence (Al) with machine learning (ML) approach has never been integrated with the hospital information system (HIS) for predicting major adverse cardiac events (MACE) in patients with chest pain in the emergency department (ED). Therefore, we conducted the present study to clarify it.

Methods: In total, 85,254 ED patients with chest pain in three hospitals between 2009 and 2018 were identified. We randomized the patients into a 70\%/30\% split for ML model training and testing. We used 14 clinical variables from their electronic health records to construct a random forest model with the synthetic minority oversampling technique preprocessing algorithm to predict acute myocardial infarction (AMI) $<1$ month and all-cause mortality $<1$ month. Comparisons of the predictive accuracies among random forest, logistic regression, support-vector clustering (SVC), and K-nearest neighbor (KNN) models were also performed.
\end{abstract}

Results: Predicting MACE using the random forest model produced areas under the curves (AUC) of 0.915 for AMl $<1$ month and 0.999 for all-cause mortality $<1$ month. The random forest model had better predictive accuracy than logistic regression, SVC, and KNN. We further integrated the Al prediction model with the HIS to assist physicians with decision-making in real time. Validation of the Al prediction model by new patients showed AUCS of 0.907 for $\mathrm{AMl}<1$ month and 0.888 for all-cause mortality $<1$ month.

Conclusions: An Al real-time prediction model is a promising method for assisting physicians in predicting MACE in ED patients with chest pain. Further studies to evaluate the impact on clinical practice are warranted.

Keywords: Artificial intelligence, Chest pain, Emergency department, Machine learning, Major adverse cardiac events

\footnotetext{
* Correspondence: chienchenghuang@yahoo.com.tw

${ }^{+}$Pei-I Zhang and Chien-Chin Hsu contributed equally to this work.

2Department of Emergency Medicine, Chi Mei Medical Center, 901

Zhonghua Road, Yongkang District, Tainan City 710, Taiwan

${ }^{11}$ Department of Senior Services, Southern Taiwan University of Science and Technology, Tainan, Taiwan

Full list of author information is available at the end of the article
}

(c) The Author(s). 2020 Open Access This article is licensed under a Creative Commons Attribution 4.0 International License, which permits use, sharing, adaptation, distribution and reproduction in any medium or format, as long as you give appropriate credit to the original author(s) and the source, provide a link to the Creative Commons licence, and indicate if changes were made. The images or other third party material in this article are included in the article's Creative Commons licence, unless indicated otherwise in a credit line to the material. If material is not included in the article's Creative Commons licence and your intended use is not permitted by statutory regulation or exceeds the permitted use, you will need to obtain permission directly from the copyright holder. To view a copy of this licence, visit http://creativecommons.org/licenses/by/4.0/ The Creative Commons Public Domain Dedication waiver (http://creativecommons.org/publicdomain/zero/1.0/) applies to the data made available in this article, unless otherwise stated in a credit line to the data. 


\section{Background}

Chest pain is one of the most common complaints that patients present with in the emergency department (ED) and accounts for 5 to $20 \%$ of all ED visits [1]. The causes of chest pain range from myalgia to potentially lifethreatening diseases, such as acute coronary syndrome (ACS), aortic dissection, or pulmonary embolism [2]. Therefore, ED physicians often face a challenge when they need to decide on a diagnosis and disposition. Meanwhile, ED physicians may arrange a series of cardiac evaluations or an imaging study to exclude the list of differential diagnoses or even liberally hospitalize patients. However, $<10 \%$ of ED patients with chest pain are eventually diagnosed with ACS, and the liberal use of serial studies and hospitalization results in a high medical cost [3]. Therefore, developing a useful clinical prediction rule to help ED physicians with decisionmaking becomes an important issue.

The HEART (History, Electrocardiography, Age, Risk Factors, Troponin) score was created in 2008 to facilitate accurate diagnostic and therapeutic choices in ED patients with chest pain [4]. The original risk stratifications and suggested dispositions according to HEART score are as follows: (1) $0-3$ points (2.5\% for major adverse cardiac events [MACE]): an immediate discharge; 4-6 points (20.3\% for MACE): implies hospitalization for clinical observation; and (3) $\geq 7$ points: ( $72.7 \%$ for MACE): early invasive strategies [4]. The HEART score has the advantages of high accuracy and has been well-validated in many studies [5-7]. The disadvantage of the HEART score is that it takes time to calculate for prediction; even a calculator is available online [8], which limits its use in the over-crowded ED. There are other scores (e.g., EDACS, TMACS, and Vancouver), which do not take substantial time to calculate [9-11]. They also force the acquisition of important data that may not otherwise be acquired. In recent years, artificial intelligence (AI), including machine learning (ML) techniques, has been used more and more often for developing prediction models and risk stratification in the ED $[12,13]$. Many studies have shown that ML outperforms traditional metrics $[12,13]$. In addition, $\mathrm{ML}$ has the advantage of handling more variables that are already available through electronic medical records (EMRs) and providing real-time feedback in terms of risk stratification to physicians if implemented in the hospital information system (HIS) [12, 13]. However, the issue about utility of ML integration with the HIS for predicting MACE in ED patients with chest pain is still unclear. We did not find any studies on this issue by searching for "acute myocardial infarction," "chest pain," "emergency department," "death," "machine learning," "major adverse cardiac events," and "mortality" in PubMed and Google Scholar. Therefore, we conducted this study intending to clarify this issue.

\section{Methods \\ Study design, setting, and participants}

We established a multi-disciplinary team including emergency physicians, data scientists, information engineers, nurse practitioners, and quality managers for this study and AI implementation. Adult patients (age $\geq 20$ years) with chest pain who visited the EDs of three hospitals (Chi Mei Medical Center, Chi Mei Liouying Hospital, and Chi Mei Chiali Hospital) between 2009 and 2018 were identified into the present study (Fig. 1). The criteria for chest pain are defined as the initial diagnostic impression according to the International Classification of Diseases, Ninth Revision, Clinical Modification (ICD-9-CM) of 786.5 or ICD-10 of R079 in the index ED visit. Patients who did not have a record of subsequent follow-up were excluded.

\section{Definitions of feature variables}

The 14 feature variables recruited for the analyzes were the suggested predictors of MACE in previous studies as follows [5, 6, 14]: age, sex, smoking, body mass index (BMI), and past histories of hypertension (ICD-9-CM: 401-405 or ICD-10: I10-I16), hyperlipidemia (ICD-9CM: 272.0-272.5, 277.7 or ICD-10: E78.0-E78.5, E88.81), diabetes (ICD-9-CM: 250 or ICD-10: E08-E13), chronic kidney disease (ICD-9-CM: 585 or ICD-10: N18), coronary artery disease (ICD-9-CM: $410-414$ or ICD-10: I20-I25), cerebrovascular diseases (ICD-9-CM: 430-438 or ICD-10: I60-I69, G45), peripheral artery occlusive disease (ICD-9-CM: 443.9 or ICD-10: I73.9), and last laboratory data including high sensitive troponin-I, hemoglobin, and serum creatinine. The last high sensitive troponin-I is automatically captured into the AI prediction model when the ED physician pushes the AI button in the HIS when they need assistance. The past histories were defined as the diagnoses before the index visit. Electrocardiography was not recruited into this study due to the technical limitation at current stage.

\section{Outcome measurements}

We defined two outcome measurements as follows: (1) acute myocardial infarction (AMI) (ICD-9-CM: 410-414 or ICD-10: I20-I25) within 1 month; and (2) all-cause mortality within 1 month after the index ED visit.

\section{Ethical statement}

This study was approved by the institutional review board of the Chi Mei Medical Center. Informed consent from the patients was waived because this study is retrospective, and it contains de-identified information, which does not affect the rights and welfare of the patients. 


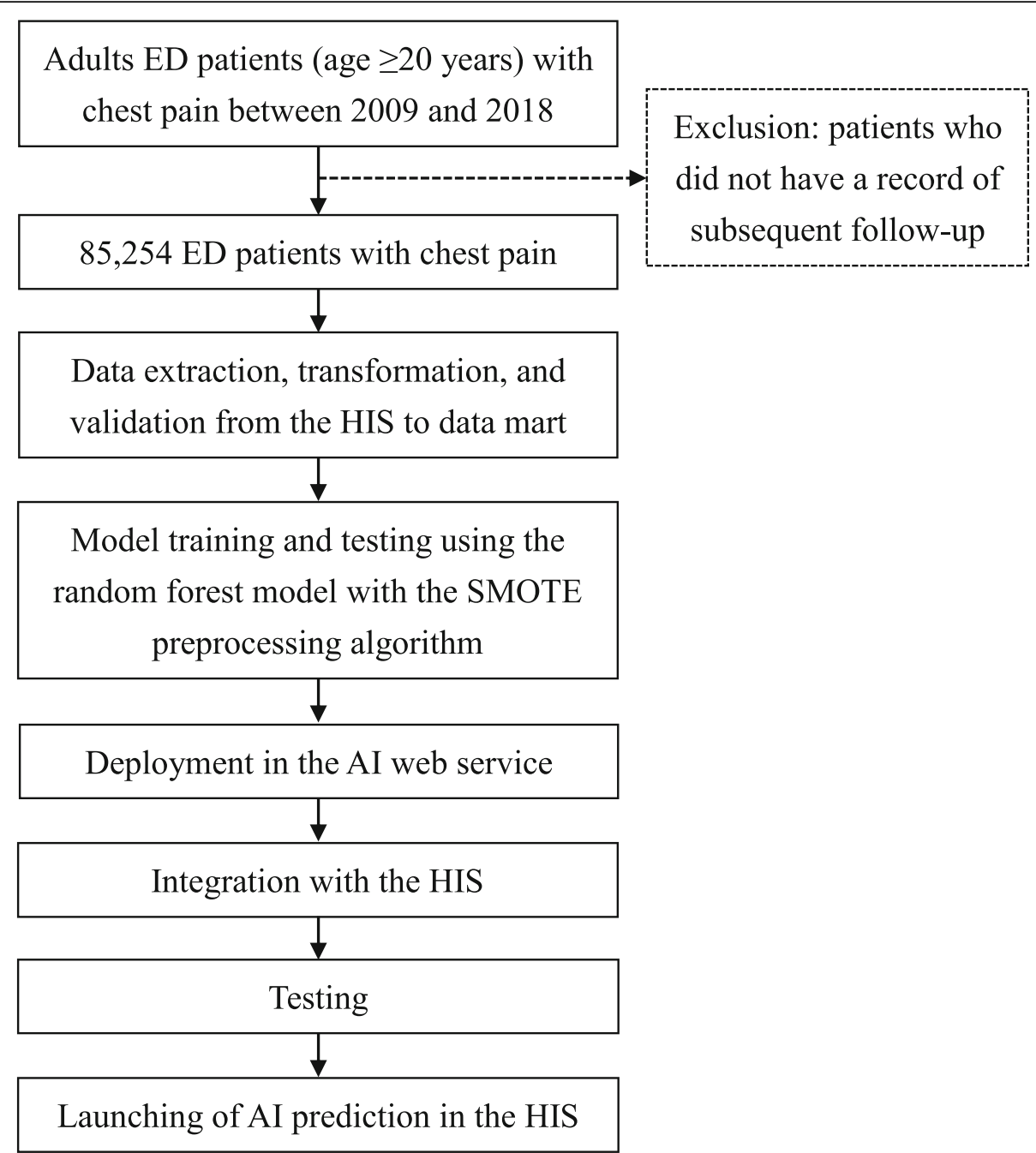

Fig. 1 Flow chart for integrating the Al prediction model for ED patients with chest pain with the HIS. Al, artificial intelligence; ED, emergency department; HIS, hospital information system; SMOTE, synthetic minority oversampling technique

Data processing, comparison, and application in the HIS First, the data were extracted from EMRs in the HIS and transformed and validated into a data mart for further analyzes. Missing and ambiguous data were defined carefully by the group meeting including emergency physicians, data scientists, information engineers, nurse practitioners, and quality managers: (1) delete the data if the feature variable could not be estimated (e.g., missing "sex") or many feature variables are missing; and (2) add average value if the missing feature variable could be estimated (e.g., missing "BMI"). Second, we used a random forest model with the synthetic minority oversampling technique (SMOTE) preprocessing algorithm to predict AMI $<1$ month and all-cause mortality $<1$ month. Third, we compared the accuracy, precision, sensitivity, specificity, F1, and area under the curve (AUC) among random forest, logistic regression, support-vector clustering (SVC), and K-nearest neighbor (KNN) models.
We divided the population into training and testing cohorts representing 70 and $30 \%$ of the patients, respectively. Fourth, we implemented the predictive model, by using random forest with SMOTE, in the HIS. After one month of testing and validation, we launched the AI prediction model in the HIS to assist ED physicians with decision-making in real time. Using the same data collection tool, we also prospectively identified new ED patients with chest pain between Jan 1, 2019 and Oct 31, 2019 after implementation of the AI prediction model to validate its accuracy.

\section{Results}

In total, 85,254 ED patients with chest pain in three hospitals between 2009 and 2018 were identified into the present study. The mean \pm standard deviation (SD) age was $57.7 \pm 17.9$ years, and the proportion of males was $55.3 \%$ (Table 1). The proportion of the three age 
Table 1 Demographic characteristics, medical histories, and adverse outcomes within one month in ED patients with chest pain

\begin{tabular}{ll}
\hline Variable & Total patients $(\boldsymbol{n}=\mathbf{8 5 , 2 5 4})$ \\
\hline Age (years) & $57.7 \pm 17.9$ \\
Age subgroup (\%) & 12.8 \\
$20-34$ & 19.5 \\
$35-49$ & 30.0 \\
$50-64$ & 37.7 \\
$\geq 65$ & \\
Sex, & 44.7 \\
Female & 55.3 \\
Male & 21.8 \\
Smoking & $24.8 \pm 3.7$ \\
BMI & \\
Medical histories (\%) & 47.6 \\
Hypertension & 21.1 \\
Hyperlipidemia & 26.2 \\
Diabetes & 8.2 \\
Chronic kidney disease & 38.3 \\
Coronary artery disease & 15.1 \\
Cerebrovascular disease & 3.6 \\
Peripheral arterial occlusion disease & \\
Laboratory data & $113.9 \pm 2603.2$ \\
High sensitive troponin-I (pg/mL) & $1.4 \pm 2.4$ \\
Hemoglobin (mg/dL) & \\
Serum creatinine (mg/dL) & \\
Outcome within one month (\%) & \\
AMl & \\
All-cause mortality & \\
\hline Dat a & \\
\hline
\end{tabular}

Data are presented as mean \pm SD or percent. $E D$ emergency department; $S D$ standard deviation; $B M I$ body mass index; $A M I$ acute myocardial infarction

subgroups were age 20-34 (12.8\%), age 35-49 (19.5\%), age $50-64(30.0 \%)$, and age $\geq 65(37.7 \%)$. The proportion of smokers was $21.8 \%$ and mean \pm SD of BMI was $24.8 \pm 3.7$. The medical histories of ED patients included hypertension (47.6\%), hyperlipidemia (21.1\%), diabetes (26.2\%), chronic kidney disease (8.2\%), coronary artery disease (38.3\%), cerebrovascular disease (15.1\%), and peripheral arterial occlusion disease (3.6\%). The mean \pm SD of high sensitive troponin-I, hemoglobin, and serum creatinine were $113.9 \pm 2603.2 \mathrm{pg} / \mathrm{mL}, 13.1 \pm 2.1 \mathrm{mg} / \mathrm{dL}$, and $1.4 \pm 2.4 \mathrm{mg} / \mathrm{dL}$, respectively. AMI $<1$ month was $20.3 \%$, and all-cause mortality $<1$ month was $0.3 \%$.

The random forest model with the SMOTE preprocessing algorithm showed that the AUCs for predicting AMI $<1$ month and all-cause mortality $<1$ month were 0.915 and 0.999 (Table 2). Tree-based estimators were used to compute the feature importance value, which helps to discard irrelevant features [15]. In our random forest model, we included all 14 feature variables based on the feature importance values (Supplement Figure 1 and Supplement Figure 2) and expert judgment. Comparisons of predictive accuracies among the random forest, logistic regression, SVC, and $\mathrm{KNN}$ revealed that the random forest model had the best AUC, accuracy, precision, sensitivity, specificity, and F1 of all the models (Table 3). In the prediction of AMI $<1$ month, SVC has the poorest AUC of 0.631. Logistic regression has the poorest AUC of 0.716 in the prediction of all-cause mortality $<1$ month. We integrated the AI prediction model with the HIS to assist the ED physicians in real-time decision-making. The time taken to generate prediction results is $<1 \mathrm{~s}$ when the ED physician pushes the AI button (Supplementary Figure 3).

We identified 3741 new ED patients with chest pain between Jan 1, 2019 and Oct 31, 2019 to validate the AI prediction model (Table 4). The AUCs of AMI < 1 month and all-cause mortality $<1$ month were 0.907 and 0.888, respectively. Between June 1, 2019 and May 31, 2020 , the use rate of AI prediction model was $12.4 \%$ with a satisfaction of $4.98 \pm 0.15$ by 5 -point Likert scale.

\section{Discussion}

We used a big-data-driven approach, ML, and integration with HIS to build a real-time prediction for MACE in ED patients with chest pain. In the prospective validation, the AI prediction model has an AUC of 0.907 for AMI $<1$ month and an AUC of 0.888 for all-cause mortality < 1 month. Compared with other ML algorithms, the random forest model had better accuracy than logistic regression, SVC, and KNN. Validation of

Table 2 Evaluation report using the random forest model with the SMOTE preprocessing algorithm on the adverse outcomes in ED patients with chest pain

\begin{tabular}{|c|c|c|c|c|c|c|c|c|c|c|}
\hline Outcome & Number & $\begin{array}{l}\text { Negative } \\
\text { outcome }\end{array}$ & $\begin{array}{l}\text { Positive } \\
\text { outcome }\end{array}$ & $\begin{array}{l}\text { Number after imbalanced } \\
\text { processing (over sampling) }\end{array}$ & Accuracy & Precision & Sensitivity & Specificity & F1 & AUC \\
\hline $\mathrm{AMl}<1$ month & 85,254 & 67,921 & 17,333 & 135,842 & 0.915 & 0.916 & 0.915 & 0.882 & 0.915 & 0.915 \\
\hline $\begin{array}{l}\text { All-cause } \\
\text { mortality < } 1 \\
\text { month }\end{array}$ & 85,254 & 85,040 & 214 & 170,080 & 0.999 & 0.999 & 0.999 & 0.999 & 0.999 & 0.999 \\
\hline
\end{tabular}

SMOTE synthetic minority oversampling technique; ED emergency department; F1, $2 \times$ (precision $\times$ recall/precision + recall); $A U C$ area under the curve; $A M I$ acute myocardial infarction 
Table 3 Comparisons of predictive accuracies among random forest, logistic regression, SVC, and KNN models for adverse outcomes of ED patients with chest pain

\begin{tabular}{|c|c|c|c|c|c|c|}
\hline Outcomes and predictive models & Accuracy & Precision & Sensitivity & Specificity & F1 & AUC \\
\hline \multicolumn{7}{|l|}{$\mathrm{AMI}<1$ month } \\
\hline Random forest & 0.915 & 0.916 & 0.915 & 0.882 & 0.915 & 0.915 \\
\hline Logistic regression & 0.868 & 0.885 & 0.868 & 0.766 & 0.867 & 0.868 \\
\hline SVC & 0.631 & 0.635 & 0.631 & 0.538 & 0.627 & 0.631 \\
\hline KNN & 0.865 & 0.880 & 0.865 & 0.766 & 0.864 & 0.865 \\
\hline \multicolumn{7}{|l|}{ All-cause mortality $<1$ month } \\
\hline Random forest & 0.999 & 0.999 & 0.999 & 1.000 & 0.999 & 0.999 \\
\hline Logistic regression & 0.716 & 0.717 & 0.716 & 0.690 & 0.716 & 0.716 \\
\hline SVC & 0.656 & 0.660 & 0.656 & 0.584 & 0.654 & 0.656 \\
\hline KNN & 0.969 & 0.971 & 0.969 & 0.940 & 0.969 & 0.969 \\
\hline
\end{tabular}

SVC support-vector clustering; KNN K-nearest neighbors; ED emergency department; F1 2 x (precision $\mathrm{x}$ recall/precision + recall); $A U C$ area under the curve; $A M I$ acute myocardial infarction

the AI prediction model using new patients also showed excellent accuracy.

Risk stratification and disposition of ED patients with chest pain is always a difficult challenge for ED physicians, especially in overcrowding situations $[6,7,16]$. A missed diagnosis of AMI is a nightmare for ED physicians; therefore, developing a clinical prediction rule becomes a useful method to assist with decision-making and disposition [16]. The Thrombolysis In Myocardial Infarction (TIMI) score was originally developed to predict adverse cardiac outcomes (death, [re]infarction, or recurrent severe ischemia requiring revascularization) within 14 days of presentation for patients with unstable angina or non-ST-segment-elevation myocardial infarction [17]. The TIMI score was also used to predict MACE in ED patients with chest pain [18]. Another famous tool, the GRACE score, was also developed in patients already diagnosed with AMI [19]. In contrast to the TIMI and GRACE scores, the HEART score was developed more specifically for rapid risk stratification and disposition in ED patients with chest pain [4]. The HEART score has the benefits of ease of recall and use, ready availability of predictors, a focus on short-term outcomes, specificity for ED management, and identification of three risk groups [20]. Compared with the TIMI and GRACE scores, the HEART score performed better to discriminate MACE in ED patients with chest pain [21].

The real-time AI prediction model in the present study has good accuracy for risk stratification in ED patients with chest pain. In addition, its features of automatic and rapid capture of the predictors from EMRs and improving ML algorithms provide ED physicians with new hope for improving care in this population. The random forest model adopted in the present study is a common algorithm for predicting outcomes and selecting predictors in the ED [13, 22]. A study reported that ML outperformed CURB-65, MEDS, and mREMS as well as traditional analytical techniques for predicting in-hospital mortality in ED patients with sepsis [22]. Another study reported that ML-based variable selection is promising method of discovering relevant and significant predictors of MACE in ED patients with chest pain [13]. In addition to the random forest model, we used the SMOTE algorithm, an oversampling approach, to adjust imbalanced data in the present study [23-25]. In the SMOTE algorithm, the minority group is over-sampled by creating synthetic examples [23-25]. The SMOTE is suggested to be better than the undersampling approach, which has the drawback of disregarding potentially useful data [23-25].

Implementation of AI prediction model in healthcare raises many new issues, including malpractice liability, insurance coverage, and EMR platforms. Legal issues include technology manufacturers' and health care professionals' liability, particularly if they can't explain the suggestions generated by AI prediction model [26]. New legal doctrine is necessary for AI-related medical malpractice [26]. For healthcare insurance, a clear and effective data governance framework is critical [27]. In the design and use of EMR and AI, legal standards need to be enacted and insurance company should be

Table 4 Validation of the Al prediction model with new ED patients with chest pain $(n=3741)$

\begin{tabular}{|c|c|c|c|c|c|c|}
\hline Outcome & Accuracy & Precision & Sensitivity & Specificity & F1 & $\overline{A U C}$ \\
\hline AMl $<1$ month & 0.907 & 0.908 & 0.929 & 0.885 & 0.907 & 0.907 \\
\hline All-cause mortality $<1$ month & 0.888 & 0.908 & 0.775 & 0.999 & 0.886 & 0.888 \\
\hline
\end{tabular}

$E D$ emergency department; F1, $2 \times$ (precision $\times$ recall/precision + recall); $A U C$ area under the curve; $A M I$ acute myocardial infarction 
encouraged to adopt a human-centered approach [27]. Regulators and policy-makers and insurance company need to work together to ensure that the big-data driven AI are transparent and accurate [27].

The major strength of this study is that we developed the first real-time AI prediction model integrated with the HIS for predicting MACE in ED patients with chest pain. Another strength was that we identified new patients to validate the AI prediction model. The limitations are as follows. First, we did not include electrocardiography in the analyzes due to the difficultly of interpreting unstructured data. However, our AI prediction model using structured data only performed well. Further studies by including electrocardiography may be needed to evaluate the additional value. Second, ML has the problems of interpretability and inferences about variables [28]. However, we believe that the random forests used in this study provide for good interpretability through the feature importance plots and the individual trees. Third, some data were not available due to the retrospective nature of this study. We used expert consultation to decide how to manage missing values. Fourth, we did not evaluate the impact of the AI prediction model on clinical practice. Although it is beyond the scope of this study, it may be necessary to evaluate the impact on physicians' decisions, including acceptance and rejection, skepticism, concerns, change their clinical practices, and patient outcomes. Fifth, we used ICD-9-CM of chest pain to recruit the patients into this study, which may exclude patients who presented with chest pain but had, for instance, AMI. However, patients with immediate diagnosis of AMI are not the target population for the AI prediction model (e.g., significant ST elevation on the electrocardiography). The main difficulty is evaluating the patients with equivocal finding in the initial presentation. Therefore, the AI prediction model may play the important role to assist ED physicians for making decision in such a circumstance. Sixth, the AI prediction model may not be generalizable to other hospitals. However, this study was supposed to be a proof of concept of using AI for this purpose. We also suggest the same algorithm might be used at other institutions. Re-training and testing in other hospitals are suggested to overcome this issue.

\section{Conclusions}

We integrated the first real-time AI prediction model with the HIS to predict short-term MACE in ED patients with chest pain. The AI prediction model has excellent accuracy and a rapid response for assisting ED physicians in decisions and disposition. Validation on new patients also showed excellent performance. Further studies about the impact of an AI prediction model on clinical practice and outcomes, as well as including electrocardiography in the analyzes, may be needed.

\section{Supplementary information}

Supplementary information accompanies this paper at https://doi.org/10. 1186/s13049-020-00786-X.

Additional file 1: Supplementary Figure 1. Feature importance according to a random forest model for predicting $\mathrm{AMl}<1$ month in ED patients with chest pain. AMI, acute myocardial infarction; ED, emergency department.

Additional file 2: Supplementary Figure 2. Feature importance according to a random forest model for predicting all-cause mortality < 1 month in ED patients with chest pain. AMI, acute myocardial infarction; ED, emergency department.

Additional file 3: Supplementary Figure 3. Screenshot of the realtime Al prediction model in the HIS for predicting MACE in ED patients with chest pain. Al, artificial intelligence; HIS, hospital information system; MACE, major adverse cardiac events; ED, emergency department.

\section{Abbreviations}

Al: Artificial intelligence; ML: Machine learning; HIS: Hospital information system; MACE: Major adverse cardiac events; ED: Emergency department; AMI: Acute myocardial infarction; SVC: Support-vector clustering; KNN: Knearest neighbor; AUC: Areas under the curves; ACS: Acute coronary syndrome; HEART: History, Electrocardiography, Age, Risk Factors, Troponin; EMRs: Electronic medical records; ICD-9-CM: International Classification of Diseases, Ninth Revision, Clinical Modification; BMl: Body mass index; SMOTE: Synthetic minority oversampling technique; SD: Standard deviation; TIMI: Thrombolysis In Myocardial Infarction

\section{Acknowledgements}

We thank Enago for the English revision.

\section{Authors' contributions}

PIZ, CC Hsu, CF Liu, and CC Huang designed and conceived this study and wrote the manuscript. CJ Chen and TL Liu performed the data processing, deployment in Al web service, integration with HIS, testing the application, and launching the application in the HIS. TL Liu performed model training and testing and statistical analysis. YK, YW Kuo, SL Hsu, HJL, and JJ Wang provided professional discussions and suggestions and wrote the manuscript. All authors read and approved the final manuscript.

\section{Funding}

This work was supported by Chi Mei Medical Center, [Grant number CMFHR CMFHR10991]

\section{Availability of data and materials}

Due to the nature of this research, participants of this study did not agree for their data to be shared publicly, so supporting data is not available.

\section{Ethics approval and consent to participate}

This study was approved by the institutional review board of the Chi Mei Medical Center. Informed consent from the patients was waived because this study is retrospective, and it contains de-identified information, which does not affect the rights and welfare of the patients.

\section{Consent for publication}

Not applicable.

\section{Competing interests}

All authors denied any financial and non-financial competing interests.

\section{Author details}

${ }^{1}$ Department of Emergency Medicine, Chi Mei Medical Center, Liouying, Tainan, Taiwan. ${ }^{2}$ Department of Emergency Medicine, Chi Mei Medical Center, 901 Zhonghua Road, Yongkang District, Tainan City 710, Taiwan. ${ }^{3}$ Department of Biotechnology, Southern Taiwan University of Science and Technology, Tainan, Taiwan. ${ }^{4}$ Graduate Institute of Medical Sciences, College of Health Sciences, Chang Jung Christian University, Tainan, Taiwan. ${ }^{5}$ Information Systems, Chi Mei Medical Center, Tainan, Taiwan. ${ }^{6}$ Center for Quality Management, Chi Mei Medical Center, Tainan, Taiwan. ${ }^{7}$ Department 
of Nursing, Chi Mei Medical Center, Tainan, Taiwan. ${ }^{8}$ Department of Emergency Medicine, Taipei Medical University, Taipei, Taiwan. ${ }^{9}$ Department of Medical Research, Chi Mei Medical Center, 901 Zhonghua Road, Yongkang District, Tainan City 710, Taiwan. ${ }^{10}$ Allied Al Biomed Center, Southern Taiwan University of Science and Technology, Tainan, Taiwan. ${ }^{11}$ Department of Senior Services, Southern Taiwan University of Science and Technology, Tainan, Taiwan. ${ }^{12}$ Department of Environmental and Occupational Health, College of Medicine, National Cheng Kung University, Tainan, Taiwan.

Received: 28 June 2020 Accepted: 2 September 2020

Published online: 11 September 2020

\section{References}

1. Bayon Fernandez J, Alegria Ezquerra E, Bosch Genover X, Cabades O'Callaghan A, Iglesias Garriz I, Jimenez Nacher JJ, Malpartida De Torres F, Sanz Romero G, Grupo de Trabajo ad hoc de la Seccion de Cardiopatia Isquemica y Unidades Coronarias de la Sociedad Espanola de C. Chest pain units. Organization and protocol for the diagnosis of acute coronary syndromes. Rev Esp Cardiol. 2002;55(2):143-54.

2. Knockaert DC, Buntinx F, Stoens N, Bruyninckx R, Delooz H. Chest pain in the emergency department: the broad spectrum of causes. Eur J Emerg Med. 2002;9(1):25-30.

3. Pope JH, Aufderheide TP, Ruthazer R, Woolard RH, Feldman JA, Beshansky $J R$, Griffith JL, Selker HP. Missed diagnoses of acute cardiac ischemia in the emergency department. N Engl J Med. 2000;342(16):1163-70.

4. Six AJ, Backus BE, Kelder JC. Chest pain in the emergency room: value of the HEART score. Neth Heart J. 2008;16(6):191-6.

5. Stopyra JP, Riley RF, Hiestand BC, Russell GB, Hoekstra JW, Lefebvre CW, Nicks BA, Cline DM, Askew KL, Elliott SB, et al. The HEART pathway randomized controlled trial one-year outcomes. Acad Emerg Med. 2019; 26(1):41-50.

6. Mahler SA, Lenoir KM, Wells BJ, Burke GL, Duncan PW, Case LD, Herrington DM, Diaz-Garelli JF, Futrell WM, Hiestand BC, et al. Safely identifying emergency department patients with acute chest pain for early discharge. Circulation. 2018;138(22):2456-68,

7. Leite L, Baptista R, Leitao J, Cochicho J, Breda F, Elvas L, Fonseca I, Carvalho A, Costa JN. Chest pain in the emergency department: risk stratification with Manchester triage system and HEART score. BMC Cardiovasc Disord. 2015;15:48.

8. MDCalc. HEART score for major cardiac events. [https://www.mdcalc.com/ heart-score-major-cardiac-events]. Accessed 28 June 2020.

9. Than M, Flaws D, Sanders S, Doust J, Glasziou P, Kline J, Aldous S, Troughton R, Reid C, Parsonage WA, et al. Development and validation of the emergency department assessment of chest pain score and $2 \mathrm{~h}$ accelerated diagnostic protocol. Emerg Med Australas. 2014;26(1):34-44.

10. Body R, Carley S, McDowell G, Pemberton P, Burrows G, Cook G, Lewis PS, Smith A, Mackway-Jones K. The Manchester acute coronary syndromes (MACS) decision rule for suspected cardiac chest pain: derivation and external validation. Heart. 2014;100(18):1462-8.

11. Jalili M, Hejripour Z, Honarmand AR, Pourtabatabaei N. Validation of the Vancouver chest pain rule: a prospective cohort study. Acad Emerg Med. 2012;19(7):837-42.

12. Stewart J, Sprivulis P, Dwivedi G. Artificial intelligence and machine learning in emergency medicine. Emerg Med Australas. 2018;30(6):870-4.

13. Liu N, Koh ZX, Goh J, Lin Z, Haaland B, Ting BP, Ong ME. Prediction of adverse cardiac events in emergency department patients with chest pain using machine learning for variable selection. BMC Med Inform Decis Mak. 2014;14:75.

14. Reaney PDW, Elliott HI, Noman A, Cooper JG. Risk stratifying chest pain patients in the emergency department using HEART, GRACE and TIMI scores, with a single contemporary troponin result, to predict major adverse cardiac events. Emerg Med J. 2018;35(7):420-7.

15. Breiman L, Forests R. Mach Learn. 2001;45(1):5-32.

16. DeLaney MC, Neth $M$, Thomas JJ. Chest pain triage: current trends in the emergency departments in the United States. J Nucl Cardiol. 2017; 24(6):2004-11.

17. Antman EM, Cohen M, Bernink PJ, McCabe CH, Horacek T, Papuchis $G$, Mautner B, Corbalan R, Radley D, Braunwald E. The TIMl risk score for unstable angina/non-ST elevation Ml: a method for prognostication and therapeutic decision making. JAMA. 2000;284(7):835-42.
18. Pollack CV Jr, Sites FD, Shofer FS, Sease KL, Hollander JE. Application of the TIMI risk score for unstable angina and non-ST elevation acute coronary syndrome to an unselected emergency department chest pain population. Acad Emerg Med. 2006;13(1):13-8.

19. Fox KA, Eagle KA, Gore JM, Steg PG, Anderson FA, Grace, Investigators G. The global registry of acute coronary events, 1999 to 2009--GRACE. Heart. 2010;96(14):1095-101.

20. Brady W, de Souza K. The HEART score: a guide to its application in the emergency department. Turk J Emerg Med. 2018;18(2):47-51.

21. Poldervaart JM, Langedijk M, Backus BE, Dekker IMC, Six AJ, Doevendans PA, Hoes AW, Reitsma JB. Comparison of the GRACE, HEART and TIMI score to predict major adverse cardiac events in chest pain patients at the emergency department. Int J Cardiol. 2017;227:656-61.

22. Taylor RA, Pare JR, Venkatesh AK, Mowafi H, Melnick ER, Fleischman W, Hall MK. Prediction of in-hospital mortality in emergency department patients with Sepsis: a local big data-driven, machine learning approach. Acad Emerg Med. 2016;23(3):269-78.

23. Karanasiou GS, Tripoliti EE, Papadopoulos TG, Kalatzis FG, Goletsis Y, Naka KK, Bechlioulis A, Errachid A, Fotiadis DI. Predicting adherence of patients with HF through machine learning techniques. Healthc Technol Lett. 2016; 3(3):165-70.

24. Wang Y, Wu X, Chen Z, Ren F, Feng L, Du Q. Optimizing the predictive ability of machine learning methods for landslide susceptibility mapping using SMOTE for Lishui City in Zhejiang Province, China. Int J Environ Res Public Health. 2019;16(3):368.

25. Chawla NVB, Hall KW, Kegelmeyer LO, P W. SMOTE: synthetic minority oversampling technique. J Artif Intell Res. 2002;16:321-57.

26. Sullivan HR, Schweikart SJ. Are current tort liability doctrines adequate for addressing injury caused by Al? AMA J Ethics. 2019;21(2):E160-6.

27. Ho CWL, Ali J, Caals K. Ensuring trustworthy use of artificial intelligence and big data analytics in health insurance. Bull World Health Organ. 2020;98(4):263-9.

28. Taft LM, Evans RS, Shyu CR, Egger MJ, Chawla N, Mitchell JA, Thornton SN, Bray B, Varner M. Countering imbalanced datasets to improve adverse drug event predictive models in labor and delivery. J Biomed Inform. 2009;42(2): $356-64$.

\section{Publisher's Note}

Springer Nature remains neutral with regard to jurisdictional claims in published maps and institutional affiliations.

Ready to submit your research? Choose BMC and benefit from:

- fast, convenient online submission

- thorough peer review by experienced researchers in your field

- rapid publication on acceptance

- support for research data, including large and complex data types

- gold Open Access which fosters wider collaboration and increased citations

- maximum visibility for your research: over $100 \mathrm{M}$ website views per year

At BMC, research is always in progress.

Learn more biomedcentral.com/submissions 